\title{
The influences of roughness on film thickness measurements by Mueller matrix ellipsometry
}

\author{
David A. Ramsey \\ AT\&T Bell Labs, Allentown, Pennsylvania 18105-1000
}

\author{
Kenneth C. Ludema \\ Mechanical Engineering Department, University of Michigan, Ann Arbor, Michigan 48109-2125
}

(Received 4 October 1993; accepted for publication 24 April 1994)

\begin{abstract}
The accuracy of measurement of the thickness of uniform thin films on solid substrates by null ellipsometry is severely limited when the substrate is rough. It is impossible to separate these two effects experimentally with the null ellipsometer, and there is no theoretical basis or generally used model available to separate these effects. Thus, a dual rotating-compensator Mueller matrix ellipsometer has been constructed to carry out film thickness measurements on rough substrates. Measurements were made on a set of specially prepared specimens of 8630 steel, roughened by grit blasting with aluminum oxide. Grit sizes and blasting pressures were varied to produce 11 different roughness values ranging from 0.01 to $1.295 \mu \mathrm{m} R_{a}$, as measured with a stylus tracer device. Upon each of the 11 roughness groups, films of magnesium fluoride were overlaid to thicknesses of 89 , 180,254 , and $315 \mathrm{~nm}$. One set of specimens was left uncoated. Experimental results for film thickness measurements on rough surfaces matched the ideal (for smooth surfaces) form well for roughnesses up to $0.13 \mu \mathrm{m} R_{a}$, at most angles of incidence. For rougher specimens, significant deviations in results were observed for all but the largest angles of incidence. The nonideal data were attributed to the cross-polarization effects of surface geometry, and apparent depolarization. The resolution of thickness measurements was $1 \mathrm{~nm}$ for polished specimens, and decreased continuously to $10 \mathrm{~nm}$ for the roughest specimens examined.
\end{abstract}

\section{INTRODUCTION}

The practical difficulty when using the null ellipsometer for the measurement of thin films on rough surfaces is that the settings of the polarizer and analyzer for minimum light at the photometer are different for smooth than for rough surfaces of the same material. Further, there is often considerable residual light at these settings, probably because rough surfaces produce a spatial distribution of polarization states of light, some of which will pass through the analyzer. This is particularly a problem in such areas as monitoring corrosion rates and other surface changing processes, after the surface becomes "rough." No theoretical work or models suggested for handling this problem have been found to be valid. ${ }^{1}$ Thus a study of rough surfaces was done with a type of photometric ellipsometer known as the Mueller matrix ellipsometer.

\section{THE MUELLER MATRIX ELLIPSOMETER}

An automated ellipsometer was constructed following the description and theory of Azzam ${ }^{2}$ and Hauge, ${ }^{3}$ and summarized in a more general form by Hauge. ${ }^{4}$ A sketch of the optical train is shown in Fig. 1. The instrument continuously determines all four Stokes parameters of the polarized light that is incident upon a specimen surface, $\{S\}_{2}$ (see Fig. 1), after passing through a rotating compensator. It then determines the Stokes parameters, $\{S\}_{3}$, of the light that is reflected from the specimen. The description of polarization alteration properties of the surface is given as a $4 \times 4$ trans- formation matrix, $[M]$, between the two measured states of polarized light, known as the Mueller matrix. In full form

$$
\left[\begin{array}{l}
S_{0} \\
S_{1} \\
S_{2} \\
S_{3}
\end{array}\right]_{3}=\left[\begin{array}{llll}
M_{00} & M_{01} & M_{02} & M_{03} \\
M_{10} & M_{11} & M_{12} & M_{13} \\
M_{20} & M_{21} & M_{22} & M_{23} \\
M_{30} & M_{31} & M_{32} & M_{33}
\end{array}\right] \times\left[\begin{array}{c}
S_{0} \\
S_{1} \\
S_{2} \\
S_{3}
\end{array}\right]_{2}
$$

A time-varying irradiance arrives at the photometer, provided by the rotating wave plates, and is comprised of 12 distinct harmonic frequencies superimposed on some steady (dc) signal. Fourier analysis of the signal yields 25 coefficients, which in turn are used to (over) determine the 16 elements of the specimen Mueller matrix.

The newly constructed instrument has many subsystems in which inaccuracies could adversely influence the results: Defects in the components in the optical train are readily compensated by calculation. Data are obtained when the angle of incidence is $90^{\circ}$, i.e., with no specimen in place. A typical Mueller matrix for this case is shown in Table I. The magnitude of the accumulated defect parameters is seen in the magnitude of departure of the Mueller matrix numbers in Table I from the ideal values in Eq. (4). However, the defects in the optical train are readily "backed out" in two steps. The first is to calculate the individual element defect parameters from the data in Table I. In the second step, during data acquisition from real specimens those same element defects are accounted for, i.e., "subtracted from" the specimen data. There are yet several system defects that cannot be removed by this method. These include effects due to inaccuracies in 


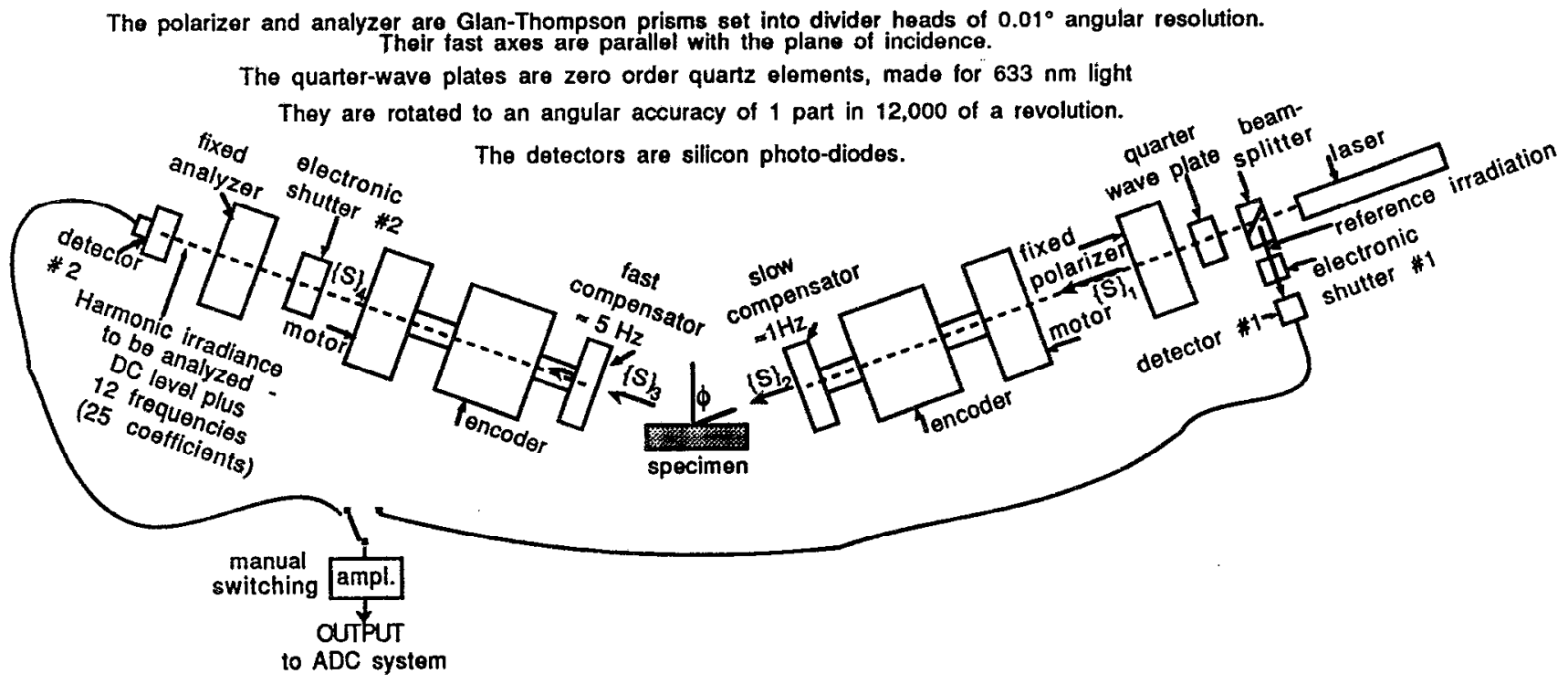

FIG. 1. Optics configuration of the Mueller matrix ellipsometer.

setting the angle of incidence, the effect of these errors in directing the light to different locations on the photometer sensing plane, instabilities in the intensity and polar state of the laser, errors in reading the angular position of the compensators, and perhaps more. The magnitude of these are best evaluated by comparison of data from the new instrument with that from a top quality null ellipsometer.

\section{VALIDATION OF THE MUELLER MATRIX ELLIPSOMETER}

Several specimens were prepared and data from these were taken with both the null ellipsometer (research instrument by Rudolph) and the new instrument. The data derived from the two instruments do not correspond exactly with each other in form. From the null ellipsometer values of $\Delta$ and $\psi$ are obtained, from which one calculates the reflection coefficients, $\rho$, in the equations of ellipsometry. ${ }^{5}$

$$
\rho=\frac{\left(R_{p} / R_{s}\right) e^{i\left[\left(\delta_{p}\right)_{r}-\left(\delta_{s}\right)_{r}\right]}}{\left(E_{p} / E_{s}\right) e^{i\left[\left(\delta_{p}\right)_{i}-\left(\delta_{s}\right)_{i}\right]}},
$$

where $E$ and $R$ are the amplitudes of the incident $(i)$ and reflected ( $r$ ) waves, $p$ and $s$ refer to the $p$ and $s$ waves, and $\delta$ refers to the absolute phase position from which

$$
\begin{aligned}
& \frac{R_{p} / R_{s}}{E_{p} / E_{s}}=\tan \psi, \\
& \left.\left.\left[\left(\delta_{p}\right)_{r}-\delta_{s}\right)_{r}\right]-\left[\left(\delta_{p}\right)_{i}-\delta_{s}\right)_{i}\right]=\Delta .
\end{aligned}
$$

For optically ideal surfaces the results of the two systems can be connected by ${ }^{4}$

$$
[M]=\frac{r_{p}^{2}+r_{s}^{2}}{2}\left|\begin{array}{cccc}
1 & -\cos 2 \psi & 0 & 0 \\
-\cos 2 \psi & 1 & 0 & 0 \\
0 & 0 & \sin 2 \psi \cos \Delta & \sin 2 \psi \sin \Delta \\
0 & 0 & -\sin 2 \psi \sin \Delta & \sin 2 \psi \cos \Delta
\end{array}\right|
$$

where $r_{p}$ and $r_{s}$ in the "matrix multiplier" are scalar amplitude reflection coefficients and are related to the irradiance of light received by the photometer.

TABLE I. Typical "straight-through" normalized Mueller matrix, using $\lambda=632.8 \mathrm{~nm}$.

\begin{tabular}{rrrr}
\hline 1.000 & 0.0001 & 0.0065 & 0.0389 \\
-0.0001 & 1.0121 & 0.0180 & -0.0008 \\
0.0058 & -0.0197 & 1.0124 & 0.0017 \\
-0.0001 & -0.0020 & -0.0004 & 0.9990 \\
\hline
\end{tabular}

For less ideal surfaces the elements in the Mueller matrix are not exactly symmetric about the diagonal and for such cases the values of $\Delta$ and $\psi$ may be calculated as follows:

$$
\begin{aligned}
& \psi=0.5 \cos ^{-1}\{[-M(0,1)-M(1,0)] / 2\}, \\
& \Delta=\tan ^{-1}\{[M(2,3)-M(3,2)] /[M(2,2)+M(3,3)]\} .
\end{aligned}
$$

Test specimens of five materials (oxidized silicon, gold coated glass, polished aluminum, polished brass, and polished steel) were used to compare the two ellipsometers. For each comparison specimen, measurements were repeated for 
TABLE II. Comparison of null and Mueller matrix ellipsometer measurements.

\begin{tabular}{llrccc}
\hline $\begin{array}{c}\text { Specimen } \\
\text { type }\end{array}$ & $\begin{array}{c}\text { Angle of } \\
\text { inc., } \theta\end{array}$ & $\begin{array}{c}\text { Avg. } \Delta \\
\text { null(deg) }\end{array}$ & $\begin{array}{c}\text { Avg. } \Delta \\
\text { MME(deg) }\end{array}$ & $\begin{array}{c}\text { Avg. } \psi \\
\text { Null(deg) }\end{array}$ & $\begin{array}{c}\text { Avg. } \psi \\
\text { MME(deg) }\end{array}$ \\
\hline Oxidized & 60 & 235.23 & 235.39 & 29.22 & 29.37 \\
silicon & 67.5 & 266.09 & 266.30 & 30.16 & 30.34 \\
& 75 & 298.28 & 298.58 & 34.52 & 34.75 \\
Gold & 60 & 130.08 & 130.31 & 43.58 & 43.76 \\
coated & 67.5 & 111.01 & 111.25 & 43.27 & 43.45 \\
glass & 75 & 84.48 & 84.35 & 43.20 & 43.33 \\
& & & & & \\
Polished & 60 & 144.17 & 143.89 & 40.99 & 40.99 \\
aluminum & 67.5 & 129.22 & 128.85 & 39.80 & 39.84 \\
& 75 & 105.31 & 104.57 & 38.61 & 38.73 \\
Polished & 60 & 126.58 & 126.22 & 40.09 & 40.18 \\
brass & 67.5 & 107.20 & 106.67 & 39.41 & 39.51 \\
& 75 & 80.89 & 79.62 & 39.41 & 39.52 \\
Polished & 60 & 145.06 & 145.03 & 33.93 & 33.95 \\
steel & 67.5 & 128.39 & 128.01 & 30.49 & 30.48 \\
& 75 & 99.65 & 98.69 & 27.56 & 27.63 \\
\hline \hline
\end{tabular}

three physical alignments (remove and remount the specimen) of the specimens on each machine, and for three angles of incidence. Very "deep" minima were obtained with the null ellipsometer and "four-zone" averaging was used.

The averaged values for $\Delta$ and $\psi$ produced by both ellipsometers are presented in Table II. It can be seen that the $\psi$ values for the Mueller matrix ellipsometer (MME) averaged $0.28 \%$ higher and the $\Delta$ values were $0.18 \%$ lower than those for the null ellipsometer. These differences are attributed to the surfaces being less than ideal, as indicated by slight, reproducible, nonsystematic asymmetry in the Mueller matrix. The repeatability of both instruments were the same.

\section{ELLIPSOMETRY OF ROUGH SURFACES}

Specimen preparation. Specimens of conducting material, with Gaussian height distributions of asperities have been selected. The specimens were 8630 steel substrates hardened and tempered to approximately 45 Rockwell "(C)." The intent was to obtain single phase martensite, tem- pered to reduce residual stresses to a low level, without softening to the point where the stylus tracer measurements would be compromised.

The surfaces were prepared by various combinations of polishing and some solid particle erosion processes to achieve a range of Gaussian roughness from $0.01 \mu \mathrm{m} R_{a}$ (arithmetic average asperity height) to $1.295 \mu \mathrm{m} R_{a}$. By continuously rotating specimens during erosion the surface roughness was not only Gaussian but also anisotropic. Roughness was measured with a stylus tracer system using a diamond stylus with a special small tip radius of $2.5 \mu \mathrm{m}$. Average roughness values of three traces per specimen are given in Table III, and the specimen groups are loosely separated into three different families according to the final process.

The material selected for the dielectric overlaid films was magnesium fluoride, for which the real component of the index of refraction in the form deposited was measured to be $n=1.38$ at $\lambda=632.8 \mathrm{~nm}$. Films of various thicknesses were deposited on most of the steel specimens using physical vapor deposition. Four film thickness groups were planned. One group was not coated and was intended for studies of the effects of surface topography alone.

The first measurements were made on the polished and uncoated specimens by null ellipsometry and Mueller matrix ellipsometry to determine the optical properties of the steel. This was found to be $2.501-3.408 i$ (for $\lambda=632.8 \mathrm{~nm}$ ), which compares well with $2.485-3.433 i$ found in Ref. 6 . The small differences are probably due to differences in microstructure and oxide film between the materials used in Ref. 6 and the present work. Next the polished and coated specimens were used to determine the thickness of the $\mathrm{MgF}_{2}$ films in the four coated groups. Using $n=1.38$ for $\mathrm{MgF}_{2}$ the film thicknesses for the four groups was found to be 89,180 , 254 , and $315 \mathrm{~nm}$. Since all specimens of various roughnesses within one film thickness group were coated simultaneously it was assumed that the rough specimens had the same film thickness of $\mathrm{MgF}_{2}$ as the smooth (polished) specimens. Finally, 55 specimens were selected for study with the identity codes given in Table IV.

For the 55 specimens, ellipsometer measurements were made in the specular direction for angles of incidence ranging from $45^{\circ}$ to $85^{\circ}$. Values for $\Delta$ and $\psi$ as calculated from selected Mueller matrices are presented in a series of graphs.

TABLE III. The surface process sequence and final roughness of specimens.

\begin{tabular}{|c|c|c|c|c|c|}
\hline & \multicolumn{3}{|c|}{ Group } & \multirow{2}{*}{$\frac{R_{a}(\mu \mathrm{m})}{0.010}$} & \multirow{2}{*}{$\frac{\text { Designation }}{\text { "Polished" }}$} \\
\hline & polished, & nde A & 1 & & \\
\hline All specimens were & & using a & 2 & 0.034 & \\
\hline processed by a series & eroded & water & 3 & 0.048 & "Intermediate \\
\hline of abrasives, & by $\mathrm{Al}_{2} \mathrm{O}_{3}$ & básed & 4 & 0.092 & roughness" \\
\hline $\begin{array}{l}\text { ending with oil based } \\
\text { polishing slurry }\end{array}$ & $\begin{array}{l}\text { of } \\
\text { various }\end{array}$ & $\begin{array}{l}\text { slurry } \\
\text { erosion }\end{array}$ & 5 & 0.127 & \\
\hline containing $6 \mu \mathrm{m}$ & grit & by & 6 & 0.256 & \\
\hline diamond particles, & sizes & air-borne & 7 & 0.492 & "Significant \\
\hline which produced & & particles & 8 & 0.557 & roughness" \\
\hline surfaces of $\approx 0.02$ & & & 9 & 0.798 & \\
\hline$\mu \mathrm{mI} R_{a}$, followed by & & & 10 & 1.002 & \\
\hline the processes listed & & & 11 & 1.295 & \\
\hline
\end{tabular}


TABLE IV. Array of specimens by film thickness and surface roughness.

\begin{tabular}{|c|c|c|c|c|c|c|}
\hline & Set A & Set $B$ & Set $C$ & Set D & Set $E$ & \\
\hline Group (film) & none & $89 \mathrm{~nm}$ & $180 \mathrm{~nm}$ & $254 \mathrm{~nm}$ & $315 \mathrm{~nm}$ & \\
\hline 1. $R_{a}=0.010 \mu \mathrm{m}$ & $1 \mathrm{~A}$ & 1B & $1 \mathrm{C}$ & ID & IE & Polished: \\
\hline 2. $R_{a}=0.034 \mu \mathrm{m}$ & $2 \mathrm{~A}$ & 2B & $2 \mathrm{C}$ & $2 \mathrm{D}$ & $2 \mathrm{E}$ & inter- \\
\hline 3. $R_{a}=0.048 \mu \mathrm{m}$ & $3 \mathrm{~A}$ & 3B & $3 \mathrm{C}$ & $3 \mathrm{D}$ & $3 E$ & mediate \\
\hline 4. $R_{a}=0.092 \mu \mathrm{m}$ & $4 \mathrm{~A}$ & $4 \mathrm{~B}$ & $4 \mathrm{C}$ & $4 \mathrm{D}$ & $4 \mathrm{E}$ & roughness \\
\hline 5. $R_{a}=0.127 \mu \mathrm{m}$ & $5 A$ & $5 B$ & $5 \mathrm{C}$ & $5 \mathrm{D}$ & $5 \mathrm{E}$ & group \\
\hline 6. $R_{a}=0.256 \mu \mathrm{m}$ & $6 \mathrm{~A}$ & $6 \mathrm{~B}$ & $6 \mathrm{C}$ & $6 \mathrm{D}$ & $6 \mathrm{E}$ & \\
\hline 7. $R_{a}=0.492 \mu \mathrm{m}$ & $7 \mathrm{~A}$ & $7 B$ & $7 \mathrm{C}$ & $7 \mathrm{D}$ & $7 \mathrm{E}$ & signifi- \\
\hline 8. $R_{a}=0.557 \mu \mathrm{m}$ & $8 \mathrm{~A}$ & $8 \mathrm{~B}$ & $8 \mathrm{C}$ & $8 \mathrm{D}$ & $8 \mathrm{E}$ & cant \\
\hline 9. $R_{a}=0.798 \mu \mathrm{m}$ & $9 \mathrm{~A}$ & $9 \mathrm{~B}$ & $9 \mathrm{C}$ & $9 \mathrm{D}$ & $9 \mathrm{E}$ & roughness \\
\hline 10. $R_{a}=1.002 \mu \mathrm{m}$ & $10 \mathrm{~A}$ & $10 \mathrm{~B}$ & $10 \mathrm{C}$ & $10 \mathrm{D}$ & $10 \mathrm{E}$ & group \\
\hline 11. $R_{a}=1.295 \mu \mathrm{m}$ & $11 \mathrm{~A}$ & 11B & $11 \mathrm{C}$ & 11D & $11 \mathrm{E}$ & \\
\hline
\end{tabular}

\section{RESULTS}

\section{A. Mueller matrices for rough surfaces}

In Table $\mathrm{V}$ typical experimental matrices are presented for a polished specimen with the $315 \mathrm{~nm}$ film of $\mathrm{MgF}_{2}$, measured at angles of incidence, $45^{\circ}, 60^{\circ}$, and $75^{\circ}$. The experimental matrices are acceptably near the ideal form given previously.

The magnitude of the matrix multiplier increases somewhat with increasing angle of incidence, and is in each case a significant fraction of 1.0 as compared with the values observed for rougher surfaces. Also, though it is a small difference in this instance, the matrix corresponding to the largest angle of incidence is slightly closer to matching the ideal form than the others [particularly in the $(2,2),(3,3)$ equality]. Again, this effect is more pronounced for rougher specimens.

In Tables VI-VIII, typical Mueller matrices are presented for specimens of increasing roughness, all with 315 $\mathrm{nm}$ films. The rougher the surface the farther the Mueller matrix departs from ideal at low angle of incidence, and the lower is the matrix multiplier. But all of the matrices approach the ideal at high angles of incidence and the matrix multipliers also become larger.

TABLE V. Typical Mueller matrices-specimen $1 E$ [roughness=polished $\left(0.01 \mu \mathrm{m} R_{a}\right)$ with $315 \mathrm{~nm}$ film $\left.(\lambda=632.8 \mathrm{~nm})\right]$.

\begin{tabular}{|c|c|c|c|c|}
\hline \multicolumn{4}{|c|}{ Angle of incidence $=45^{\circ}$} & \multirow{2}{*}{$\begin{array}{c}\text { (matrix } \\
\text { multiplier) }\end{array}$} \\
\hline 1.0000 & -0.1535 & -0.0069 & -0.0009 & \\
\hline-0.1537 & 0.9988 & 0.0714 & -0.0157 & \\
\hline-0.0063 & 0.0692 & -0.8498 & 0.4942 & $\times 0.3523$ \\
\hline 0.0014 & 0.0187 & -0.4964 & -0.8565 & \\
\hline \multicolumn{5}{|c|}{ Angle of incidence $=60^{\circ}$} \\
\hline 1.0000 & -0.3264 & -0.0167 & 0.0003 & \\
\hline-0.3268 & 0.9980 & 0.0620 & -0.0228 & $\times 0.4072$ \\
\hline 0.0117 & 0.0557 & -0.6319 & 0.6936 & \\
\hline 0.0020 & 0.0263 & -0.6920 & -0.6451 & \\
\hline \multicolumn{5}{|c|}{ Angle of incidence $=75^{\circ}$} \\
\hline 1.0000 & -0.6106 & -0.0285 & 0.0017 & \\
\hline-0.6110 & 0.9983 & 0.0537 & -0.0238 & $\times 0.4291$ \\
\hline-0.0182 & 0.0401 & -0.2600 & 0.7462 & \\
\hline 0.0018 & 0.0294 & -0.7459 & -0.2624 & \\
\hline
\end{tabular}

\section{B. $\Delta$ and $\psi$ for polished specimens}

Values of $\Delta$ and $\psi$, calculated from Mueller matrices for specimens 1A through 1E are presented in Figs. 2(a) and 2(b). These plots show the effects of increasing film thickness on near-ideal smooth substrates. The data points produce smooth curves which progress in an ordered, though seemingly cyclic, fashion with increasing film thickness. Calculations for all curves from optical properties of the materials produce similar results, i.e., differences no greater than $0.04^{\circ}$.

\section{C. $\Delta$ and $\psi$ for specimens with roughness}

The influence of a moderate roughness may be seen for specimens 5A through 5E with a roughness of $0.127 \mu \mathrm{m} R_{a}$, shown in Figs. 3(a) and 3(b). In these plots, the most notable features are:

(i) There is little reduction in ability to resolve film thickness as roughness increases.

(ii) The 0 and $315 \mathrm{~nm}$ specimens always exhibit similar results in the smooth surface case, though with in-

TABLE VI. Typical Mueller matrices-specimen 5E [roughness $=0.127 \mu \mathrm{m}$ $R_{a}, 315 \mathrm{~nm}$ film $\left.(\lambda=632.8)\right]$.

\begin{tabular}{crrrc}
\hline \hline \multicolumn{2}{c}{ Angle of incidence $=45^{\circ}$} & & & (matrix \\
1.0000 & -0.4090 & -0.0964 & 0.0582 & multiplier) \\
-0.4155 & 0.9346 & 0.2142 & -0.1554 & \\
-0.1069 & 0.2683 & -0.5133 & 0.6524 & $\times 0.1255 \times 10^{-3}$ \\
-0.0019 & 0.0563 & -0.6779 & -0.5702 & \\
& & & & \\
Angle of incidence $=60^{\circ}$ & & & \\
1.0000 & -0.7476 & -0.0396 & -0.0030 & \\
-0.7486 & 0.9992 & 0.0475 & -0.0258 & $\times 0.2589 \times 10^{-2}$ \\
-0.0394 & 0.0467 & 0.0237 & 0.6605 & \\
0.0051 & 0.0261 & -0.6621 & 0.0203 & \\
& & & & \\
Angle of incidence $=75^{\circ}$ & & & \\
1.0000 & -0.6281 & -0.0303 & -0.0028 & \\
-0.6305 & 0.9992 & 0.0190 & -0.0054 & $\times 0.4811 \times 10^{-1}$ \\
-0.0260 & 0.0077 & 0.7075 & 0.3181 & \\
0.0019 & 0.0098 & -0.3243 & -0.7077 & \\
\hline \hline
\end{tabular}


TABLE VII. Typical Mueller matrices-specimen $8 \mathrm{E}$ [roughness $=0.557$ $\mu \mathrm{m} R_{a}$ with $315 \mathrm{~nm}$ film $\left.(\lambda=632.8 \mathrm{~nm})\right]$.

\begin{tabular}{rrrrl}
\hline \hline \multicolumn{2}{c}{ Angle of incidence $=45^{\circ}$} & & & (matrix \\
1.0000 & -0.0803 & -0.1410 & 0.1310 & (multiplier) \\
-0.1519 & 0.4534 & 0.3682 & -0.2354 & \\
-0.1509 & 0.4932 & -0.2845 & 0.2218 & $\times 0.3260 \times 10^{-5}$ \\
-0.0395 & 0.0414 & -0.3633 & -0.5671 & \\
& & & & \\
\multicolumn{2}{l}{ Angle of incidence $=60^{\circ}$} & & & \\
1.0000 & -0.5261 & -0.1205 & 0.1279 & \\
-0.4131 & 0.5508 & 0.1924 & -0.2762 & $\times 0.5511 \times 10^{-5}$ \\
0.3908 & 0.5560 & -0.0417 & 0.2983 & \\
-0.0498 & 0.0923 & -0.4381 & -0.2684 & \\
& & & & \\
Angle of incidence $=75^{\circ}$ & & & \\
1.0000 & -0.6187 & 0.0288 & 0.0205 & \\
-0.6136 & 0.9640 & -0.0888 & -0.0507 & $\times 0.3257 \times 10^{-4}$ \\
-0.1215 & 0.1725 & 0.6848 & 0.1839 & \\
-0.0064 & 0.0258 & -0.1930 & 0.6735 & \\
\hline \hline
\end{tabular}

creasing roughness the $\Delta$ curves no longer intersect, and the intersection of the $\psi$ curves shifts leftward with roughness.

(iii) The plots for the $254 \mathrm{~nm}$ film specimens show a fairly abrupt change in $\Delta$ at an angle of incidence in the range of $65^{\circ}$ to $70^{\circ}$, along with a corresponding deep valley for $\psi$. Also, the $\Delta$ curves rise from $160^{\circ}$ to $360^{\circ}$ in two of the roughness groups, but decrease from $160^{\circ}$ to near $0^{\circ}$ for the other three. These trends are seen in the results of the calculation using optical properties, though accurate values of the latter are not available for the case of high roughness.

Data for rougher specimens produce a different effect. A typical example is shown for specimens $6 \mathrm{~A}$ through $6 \mathrm{E}$, all with $0.256 \mu \mathrm{m} R_{a}$, with various film thicknesses, in Figs. 4(a) and 4(b). Observations from all curves include:

(i) The plots of $\Delta$ versus angle of incidence show a good ability to differentiate between the various films (except $0.315 \mathrm{~nm}$ ), though not at large angles of incidence.

TABLE VIII. Typical Mueller matrices specimen 11E [roughness $=1.295$ $\mu \mathrm{m} R_{a}$ with $315 \mathrm{~nm}$ film $\left.(\lambda=632.8 \mathrm{~nm})\right]$.

\begin{tabular}{rrrrc}
\hline \multicolumn{7}{l}{ Angle of incidence $=45^{\circ}$} & & & \multicolumn{1}{c}{ (matrix } \\
1.0000 & -0.0366 & -0.1000 & 0.0849 & multiplier) \\
-0.1054 & 0.4462 & 0.3653 & -0.1954 & $\times 0.3639 \times 10^{-5}$ \\
-0.1175 & 0.4438 & -0.2825 & 0.2467 & \\
0.0127 & 0.0632 & 0.3427 & -0.5619 & \\
& & & & \\
Angle of incidence $=60^{\circ}$ & & & \\
1.0000 & -0.2974 & -0.1470 & 0.1253 & \\
-0.2758 & 0.5039 & 0.2396 & -0.3269 & $\times 0.4691 \times 10^{-5}$ \\
-0.2686 & 0.5154 & -0.0881 & 0.3481 & \\
-0.0009 & 0.0733 & -0.5202 & -0.3412 & \\
& & & & \\
Angle of incidence $=75^{\circ}$ & & & \\
1.0000 & -0.5528 & 0.0282 & 0.0929 & \\
-0.4494 & 0.6591 & -0.1713 & -0.2056 & $\times 0.9654 \times 10^{-5}$ \\
-0.3801 & 0.5218 & 0.3477 & 0.2180 & \\
-0.0178 & 0.0556 & -0.3277 & 0.3194 & \\
\hline \hline
\end{tabular}
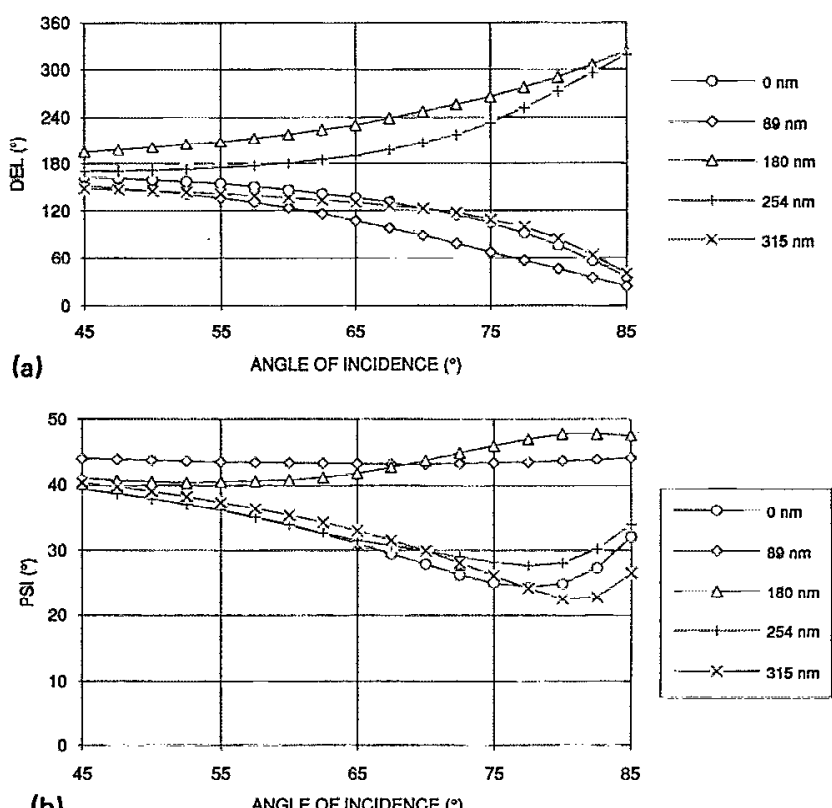

(b)

FIC. 2. (a) Experimental variation of $\Delta$ with angle of incidence for $\mathrm{MgF}_{2}$ on steel, film thickness as indicated, polished substrate $(\lambda=632.8 \mathrm{~nm})$. (b) $\mathrm{Ex}$ perimental variation of $\psi$ with angle of incidence for $\mathrm{MgF}_{2}$ on steel; film thickness as indicated, polished substrate $(\lambda=632.8 \mathrm{~nm})$.

(ii) The curves for both $\Delta$ and $\psi$ become increasingly erratic in form with increasing roughness.

(iii) It is considerably more difficult to differentiate one film from another on the basis of $\psi$ alone. [The curves, not shown here, are fairly well ordered through the eighth roughness group $\left(0.557 \mu \mathrm{m} R_{a}\right)$, but become quite erratic for subsequent groups].
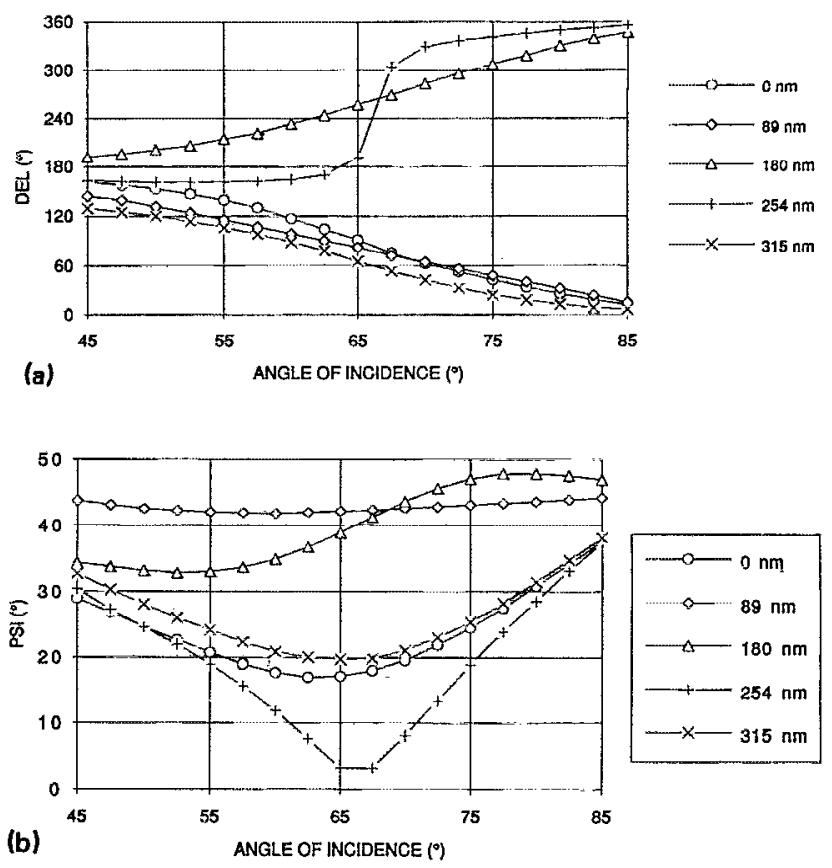

FIG. 3. (a) Experimental variation of $\Delta$ with angle of incidence for $\mathrm{MgF}_{2}$ on steel; film thickness as indicated, substrate roughness $=0.127 \mu \mathrm{m} R_{a}$ ( $\lambda$ $=632.8 \mathrm{~nm}$ ). (b) Experimental variation of $\psi$ with angle of incidence for $\mathrm{MgF}_{2}$ on steel; film thickness as indicated, substrate roughness $=0.127 \mu \mathrm{m}$ $R_{a}(\lambda=632.8 \mathrm{~nm})$. 

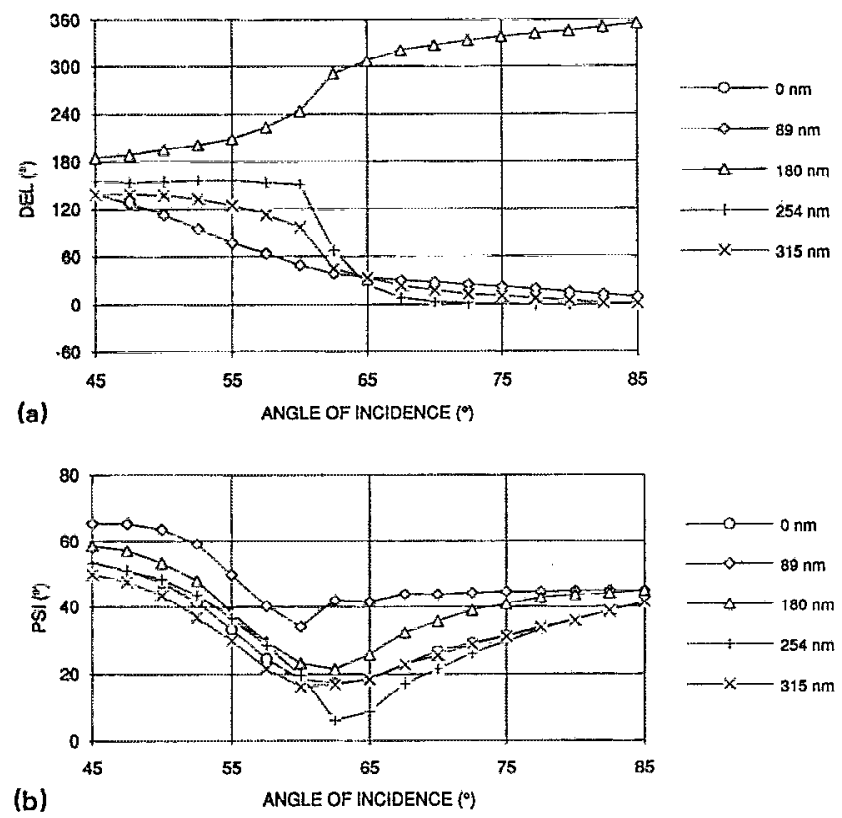

FIG. 4. (a) Experimental variation of $\Delta$ with angle of incidence for $\mathbf{M g F}_{2}$ on steel; film thickness as indicated, substrate roughness $=0.256 \mu \mathrm{m} R_{a}(\lambda$ $=632.8 \mathrm{~nm}$ ). (b) Experimental variation of $\psi$ with angle of incidence for $\mathrm{MgF}_{2}$ on steel; film thickness as indicated, substrate roughness $=0.256 \mu \mathrm{m}$ $R_{a}(\lambda=632.8 \mathrm{~nm})$.

(iv) In all cases, trends in the data are clearly evident, even if individual data points are scattered.

The data have also been plotted by comparing roughness effects for each tilm thickness group. Figures 5(a) and 5(b) are typical plots for $254 \mathrm{~nm}$ film. In viewing these plots, it is seen that
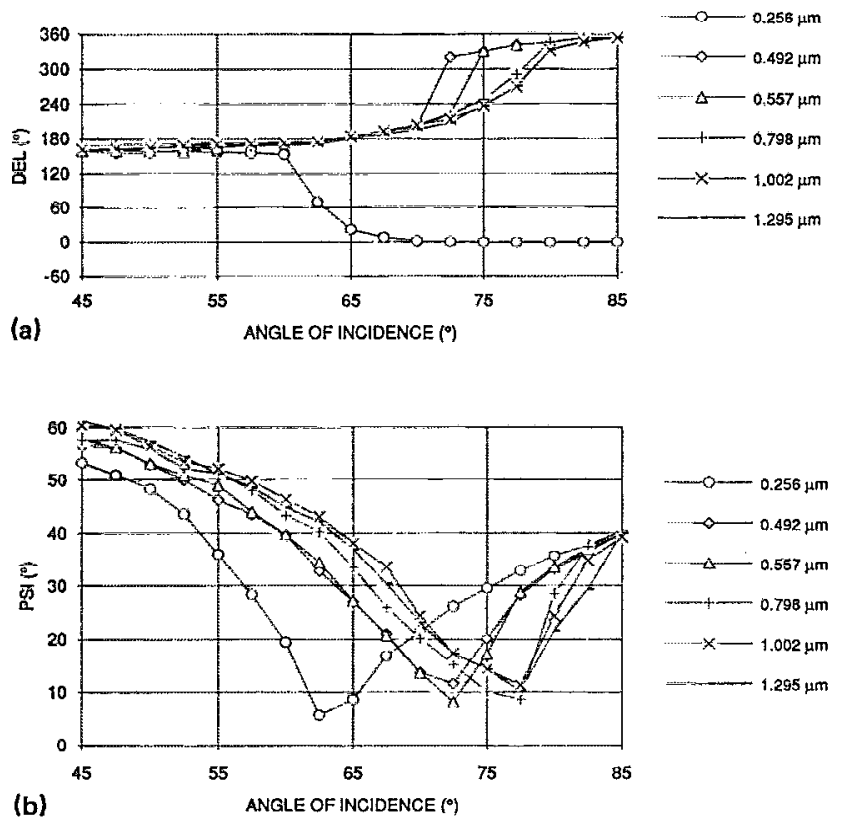

FIG. 5. (a) Experimental variation of $\Delta$ with angle of incidence for $\mathrm{MgF}_{2}$ on stecl; substrate roughness as indicated, film thickness $=254 \mathrm{~nm}(\lambda=632.8$ nm). (b) Experimental variation of $\psi$ with angle of incidence for $\mathrm{MgF}_{2}$ on steel; substrate roughness as indicated, film thickness $=254 \mathrm{~nm}(\lambda=632.8$ $\mathrm{nm})$. (i) There is a marked jump from the curves for the "intermediate" roughness category [see, e.g., Figs. 3(a) and 3(b)], to those of the second. It should be noted, though, that there is a significant increase in roughness, from 0.256 to $0.492 \mu \mathrm{m}$.

(ii) In all of the plots, there is an observable, albeit erratic, progression of results with increasing substrate roughness.

(iii) In Fig. 5(a), it is seen that once again one of the 254 $\mathrm{nm}$ film specimens shows a progression for $\Delta$ rather different from the rest of the members of the coating group, attributable to minor differences in film thickness and surface conditions.

\section{Film measurement capabilities}

In the case of an ideal specimen, it is generally stated that ellipsometric measurements can reliably differentiate between, or resolve, films differing in thickness by only $1 \mathrm{~nm}$. For the polished specimen, consider Figs. 1(a) and 1(b) and note the spacing between the curves. In making experimental measurements such as these, $\psi$ is easily repeatable to better than $0.1^{\circ}$, and $\Delta$ to something on the order of $0.5^{\circ}$. Interpolating between the curves in Fig. 1, these accuracies of measurement translate to approximately $1 \mathrm{~nm}$ or less for resolution of film thickness. Figures 4(a) and 4(b) provide similar plots for the roughest specimens measured. A similar analysis shows a resolution capability on the order of $10 \mathrm{~nm}$. The ability to resolve films of different thicknesses is continuously diminished as substrate roughness increases.

This ability to resolve film thickness can be demonstrated for any number of $\Delta$ and $\psi$ pairs using these curves. An exception to this ability, however, is seen in Fig. 2 for the 0 and $315 \mathrm{~nm}$ specimens for which both $\Delta$ and $\psi$ plots cross at $70^{\circ}$. The reason is as follows. For a filmed substrate, a reflection coefficient takes the form ${ }^{6}$

$$
R=\frac{r_{01}+r_{12} e^{(-j 2 \beta)}}{1+\left(r_{01} \cdot r_{12}\right) e^{(-j 2 \beta)}},
$$

where $r_{01}$ and $r_{12}$ involve the terms listed below, and

$$
\beta=2 \pi\left(d_{1} / \lambda\right)\left(n_{1}^{2}-n_{0}^{2} \sin ^{2} \phi_{0}\right)^{1 / 2}
$$

and reflection coefficients become cyclic functions of the film thickness.

Consider the case where

$n_{0}=1.0$ (ambient, air),

$n_{1}=1.38$ (magnesium fluoride),

$\phi_{0}=70^{\circ}$ (angle of incidence),

$d_{1}=315 \mathrm{~nm}$ (film thickness),

$\lambda=633 \mathrm{~nm}$ (wavelength of light),

and the resulting $\beta=2 \pi \times 0.503$.

It is seen that $\beta$ is nearly $\pi$, producing a full cycle for the exponential terms. The same intersection of data for the 0 and $315 \mathrm{~nm}$ film is not seen at other angles, and thus the film discrimination for $315 \mathrm{~nm}$ of $\mathrm{MgF}_{2}$ is worse at $70^{\circ}$ than at any other angle. 

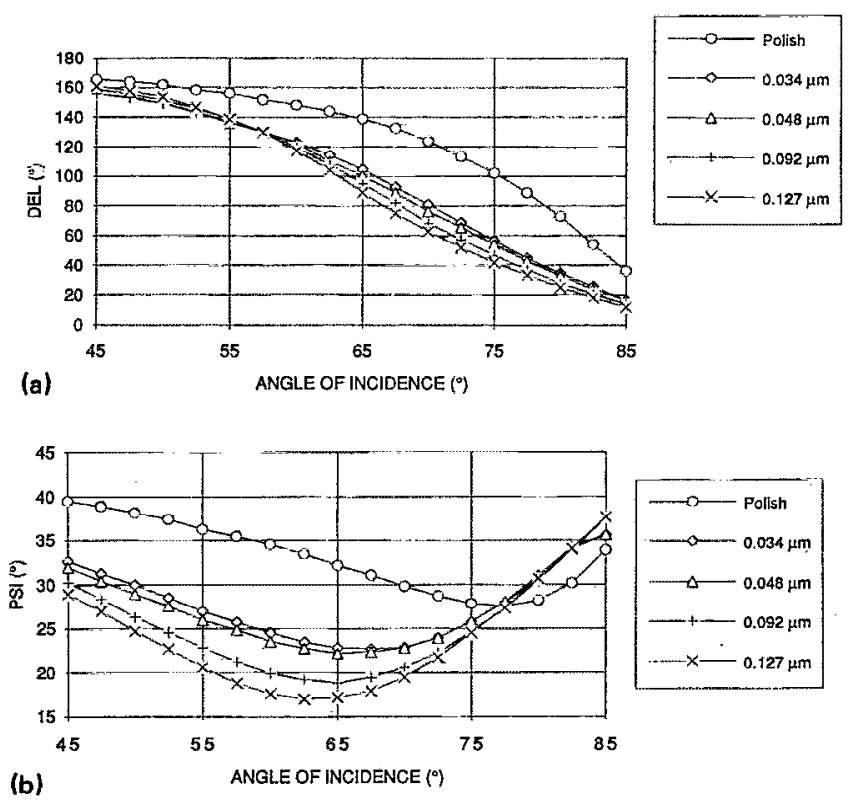

FIG. 6. (a) Experimental variation of $\Delta$ with angle of incidence for $\mathrm{MgF}_{2}$ on steel; substrate roughness as indicated, no film $(\lambda=632.8 \mathrm{~nm})$. (b) Experimental variation of $\psi$ with angle of incidence for $\mathrm{MgF}_{2}$ on steel; substrate roughness as indicated, no film $(\lambda=632.8 \mathrm{~nm})$.

\section{E. Effects of specimen preparation method}

One final point concerns the methods used for preparing the various specinens. In producing specimens for the 11 roughness categories, the only intended variable in moving from one category to the next is that of surface roughness. Other factors apparently became inadvertently involved, but did not affect the conclusions mentioned above. This is seen in the large difference between the curve for the smooth surface and those of the "intermediate" rough surfaces in Figs. 6(a) and 6(b) and may be seen between Fig. 6 and Fig. 7 as a comparison between two roughness groups. Table II shows three different preparation groups, which apparently introduced three classes of surface chemistry, corresponding to polished, water-based grit blasting, and air-based grit blasting.

\section{F. Nonideal effects}

When rough or other problem surfaces are encountered a simple $\Delta$ and $\psi$ characterization is not representative of the ideal reflection process. Other phenomena become apparent. Consider the Mueller matrix for a specimen with a roughness of $0.256 \mu \mathrm{m} R_{a}$ on which there is a film of $\mathrm{MgF}_{2} 89 \mathrm{~nm}$ thick, for an angle of incidence of $50^{\circ}$. The matrix taken from the data is

$$
\begin{aligned}
{[M]=} & 0.1633 \times 10^{-5} \\
& \times\left|\begin{array}{cccc}
1.000 & 0.1631 & -0.0322 & 0.0802 \\
0.0083 & 0.4038 & 0.2555 & -0.2158 \\
-0.0026 & 0.4297 & -0.1376 & 0.2016 \\
-0.0116 & 0.0597 & -0.3175 & -0.3690
\end{array}\right| .
\end{aligned}
$$
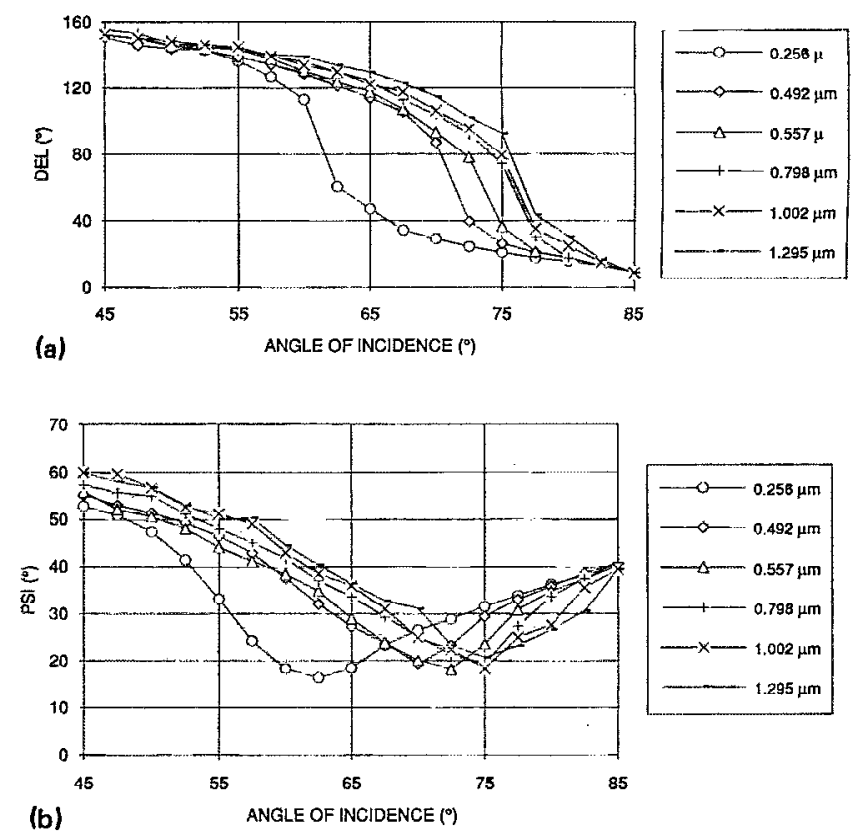

FIG. 7. (a) Experimental variation of $\Delta$ with angle of incidence for $\mathrm{MgF}_{2}$ on steel; substrate roughness as indicated, no film $(\lambda=632.8 \mathrm{~nm})$. (b) Experimental variation of $\psi$ with angle of incidence for $\mathrm{MgF}_{2}$ on steel; substrate roughness as indicated, no film $(\lambda=632.8 \mathrm{~nm})$.

The form of this matrix is far from ideal. The calculated values for $\Delta$ and $\psi$ using the equations previously given are $\Delta=314.302$ and $\psi=49.935$. Furthermore, the matrix multiplier, $0.1633 \times 10^{-5}$, is very small indicating that relatively little light is reflected in the specular direction. (The null ellipsometer results provide no perspective on the "quality" of the data.)

At least two nonideal effects can be shown in this case. First, assume that linearly " $p$ " polarized light is to be reflected from this specimen. The Stokes' vector for such an input is given by ${ }^{\top}$

$$
\{S\}_{2}=k_{1}\left|\begin{array}{l}
1 \\
1 \\
0 \\
0
\end{array}\right| \text {. }
$$

The Stokes' parameters for the reflected light may be found by taking the product of the experimental Mueller matrix with this input vector, resulting in

$$
\{S\}_{3}=k_{2}\left|\begin{array}{l}
1.1631 \\
0.4121 \\
0.4271 \\
0.0481
\end{array}\right| \text {. }
$$

Fractional polarization. The first nonideal effect is exhibited in the "degree" of polarization of the reflected light. If the reflected light were totally polarized its Stokes vector would be of numerical form such that

$$
S_{0}^{2}=S_{1}^{2}+S_{2}^{2}+S_{3}^{2} \text {. }
$$

However, from the above $\{S\}_{3}$, the total reflected irradiance, is $S_{0}^{2}=(1.1631)^{2}=1.3528$, whereas the polarized irradiance is $S_{1}^{2}+S_{2}^{2}+S_{3}^{2}=0.3546$. 
The polarization fraction is $0.3546 / 1.3528=0.262$, i.e., some $74 \%$ of the reflected irradiance is unpolarized whereas all of the incident light was polarized.

Depolarization effects have consistently been referred to as "apparent," and finally expressed as the "polarization fraction." Certainly, to the ellipsometer, some portion of the reflected light from rough surfaces has no identifiable polarization state. This probably arises from the fact that the facets of the rough surface geometry provide several different polarization states simultaneously, which is interpreted as an unpolarized component of the total irradiance.

Cross polarization. The second such effect is cross polarization. In the ideal smooth surface case, a $p$-polarized input should reflect as a $p$-polarized output (and $s$ input produces $s$ output), and the reflected output vector would have to be proportional to that of the input-which clearly it is not. In fact, this form of the output vector indicates the presence of both $p$ and $s$ polarized components in the reflected light. Thus, a cross-polarization effect is observed. (A second calculation for an " $s$ " input would show a similar effect.) As described by Beckmann, ${ }^{8,9}$ components of the electric field do act independently when they are parallel or perpendicular to the plane of incidence. In the case of a rough surface, however, there is not only a nominal plane of incidence, there are innumerable such local planes. Thus, field components which, in the global coordinate system should apparently be immune to cross polarization, intercept surface features at oblique angles, many of which arrive at the active area of the detector.

The magnitude of the cross-polarization effect can be calculated as follows. First, the Stokes' vector for the reflected light can be separated into polarized and "unpolarized" portions. Thus

$$
\{S\}_{3}=k_{2}\left(\left|\begin{array}{c}
0.5955 \\
0.4121 \\
0.4271 \\
0.0481
\end{array}\right|+\underset{\text { polarized }}{\text { unpolarized }}\right.
$$

Considering only the polarized portion of this output, the relative amplitudes of orthogonal components of the electric field vector are

$$
\begin{array}{ll}
S_{0}=E_{p}^{2}+E_{s}^{2}=0.5955, & E_{p}=0.7098, \\
S_{1}=E_{p}^{2}-E_{s}^{2}=0.4121, & E_{s}=0.3028 .
\end{array}
$$

Thus, a $p$-to-s "cross-polarization fraction" could be defined as

$$
\frac{E_{s}}{E_{p}}=\frac{0.3028}{0.7098}=0.4266
$$

It is also possible to calculate such ratios for $p$ inputs, $s$ inputs, circular inputs, etc.

\section{G. Modified Mueller matrices}

Nonideal effects notwithstanding, it is interesting to extract more realistic $\Delta$ and $\psi$ values from the experimental Mueller matrices. That is, it would be useful to have some way to separate the real optical properties of the surface material from roughness effects. Apparent depolarization effects can be "stripped" off the matrix by forcing $\{S\}_{3}$ into a totally polarized form and the specimen Mueller matrix can be altered for extraction of a more useful $\Delta$ and $\psi$. No way has been found to remove cross-polarization effects. Williams has attacked the problem by way of the Poincare representation of polarization. ${ }^{10}$ Unfortunately several other effects were found in addition to "depolarization" and cross polarization and it is not known which are independent effects.

${ }^{1}$ D. A. Ramsey, Ph.D. thesis, University of Michigan, 1985.

${ }^{2}$ R. M. A. Azzam, Opt. Lett. 2, 148 (1978).

${ }^{3}$ P. S. Hauge, J. Opt. Soc. Am. 68, 1519 (1978).

${ }^{4}$ P. S. Hauge, Surf. Sci. 96, 108 (1980).

${ }^{5}$ R. M. A. Azzam and N. M. Bashara, Ellipsometry and Polarized Light (North-Holland, Amsterdam, 1977).

${ }^{\circ}$ D. Clarke and J. F. Grainger, Polarized Light and Optical Measurement (Pergamon, Oxford, 1976).

${ }^{7}$ F. A. Jenkins and H. E. White, Fundamentals of Optics (McGraw-Hill, New York, 1976).

${ }^{8} \mathrm{P}$. Beckmann, Scattering of Electromagnetic Waves from Rough Surfaces (Macmillan, New York, 1963).

"P. Beckmann, The Depolarization of Electromagnetic Waves (Golem, Boulder, Colorado, 1968).

${ }^{10}$ M. W. Williams, Appl. Opt. 25, 3616 (1986). 